\title{
Kinematics of the Ionized Gas in the Irregular Galaxies IC 10 and NGC 4449
}

\author{
M. Rosado, A. Bullejos, M. Valdez, L. Georgiev and C. Lacey \\ Instituto de astronomía, UNAM. Apartado Postal 70-264, CP04510, \\ México, D.F., México
}

J. Borissova

Institute of Astronomy, Bulgarian Academy of Sciences, 72 Tsaridradsko chaussee, BG1784 Sofia, Bulgaria

C. Esteban

Instituto Astrofísico de Canarias, Tenerife, Canarias, España

\begin{abstract}
We have searched for supernova remnants (SNRs) in the irregular galaxies IC 10 and NGC 4449 by means of Fabry-Perot interferometry in $\mathrm{H} \alpha$ and [SII] lines, complemented by radio continuum observations. We find several new SNR candidates in each galaxy in addition to the known ones. Although these galaxies have several common properties, the distributions of SNRs in each galaxy is different. This shows that the star formation mechanisms in these galaxies act in different ways, in favor of the formation of a large nebular HII complex in IC 10, similar to 30 Dor in the Large Magellanic Cloud. We are studying the conditions over which these different star formation histories take place.
\end{abstract}

\section{Introduction}

We have selected two irregular galaxies of the Magellanic type in order to identify the supernova remnants (SNRs): IC 10 and NGC 4449. These galaxies share several properties. Here we show the results of our optical observations ( $\mathrm{H} \alpha$ and [SII] lines) using a scanning Fabry-Perot Interferometer PUMA (Rosado et al. 1995). We compare the results with radio continuum observations.

\section{Results}

IC 10 - The supernova (SN) explosions seem to be concentrated only to the south of the giant HII complex HL111 (Hodge \& Lee 1990), forming a nonthermal superbubble (Yang \& Skillman 1993). The HII complex HL111 appears to be similar to 30 Dor in the LMC but in a later stage of evolution. Figure 1 (left) shows a PUMA velocity channel image in [SII]. The circle marks the position and extent of the non-thermal radio superbubble reported by Yang \& Skillman (1993). This non-thermal superbubble has an optical counterpart 

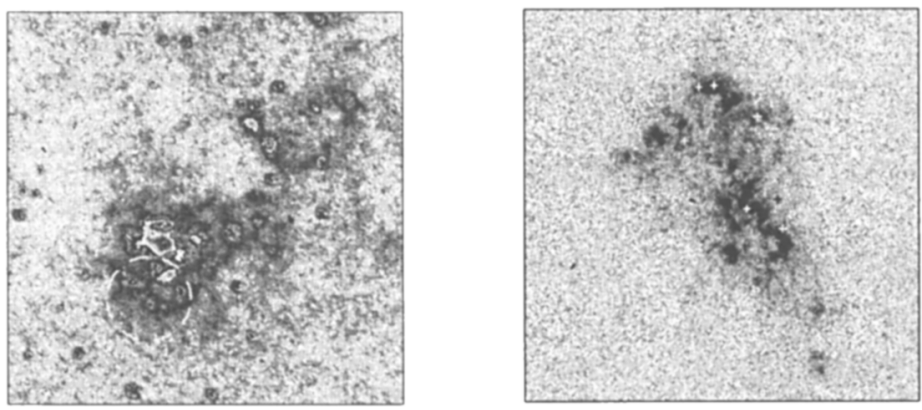

Figure 1. [SII] velocity channel images of IC 10 (left) and NGC 4449 (right). The white circle marks the non-thermal radio superbubble and the crosses mark the non-thermal radio sources.

revealed as a series of nebulosities and a curved filament running to the west of the radio superbubble over larger dimensions. Also, several of the HII regions catalogued by Hodge \& Lee (1990) are seen interconnected by faint filamentary features, forming larger shells.

NGC 4449 - Recent VLA radio continuum observations of NGC 4449 detect at least 7 non-thermal sources, including a known SNR and the nucleus of this galaxy (Lacey et al. 1998, and references therein). Figure 1 (right) shows a PUMA [SII] velocity channel image of this galaxy. We have also marked the non-thermal radio sources found by Lacey et al. (1998). All, but one, of the radio sources are embedded in optical nebulosities. We are studying the kinematics of these regions to search for optical counterparts of these non-thermal sources. Also, we have detected networks of filaments extending radially from the bar of NGC 4449. As in the case of IC 10, several of the HII regions (catalogued by Sabbadin \& Bianchini 1979) are interconnected by filaments, forming larger structures.

\section{Discussion}

We note that the distributions of SNRs are different in these galaxies. In IC 10 the SNRs seem to be concentrated near its largest HII complex, whereas in NGC 4449 the SNRs are distributed in a more uniform way. On the other hand, while in NGC 4449 it seems that the SN explosions are widely separated, in IC 10 the $\mathrm{SNe}$ combine to form larger structures. This shows that despite the several similar properties, the star formation histories of these galaxies are different.

\section{References}

Hodge, P., \& Lee, M.G. 1990, PASP, 102, 26

Lacey, C.K., et al. 1998, BAAS, 192, 5310

Rosado, M., et al. 1995, RevMexAA Conf. Ser., 3, 268

Sabbadin, F., \& Bianchini, A. 1979, PASP, 91, 280

Yang, H., \& Skillman, E.D. 1993, AJ, 106, 1448 


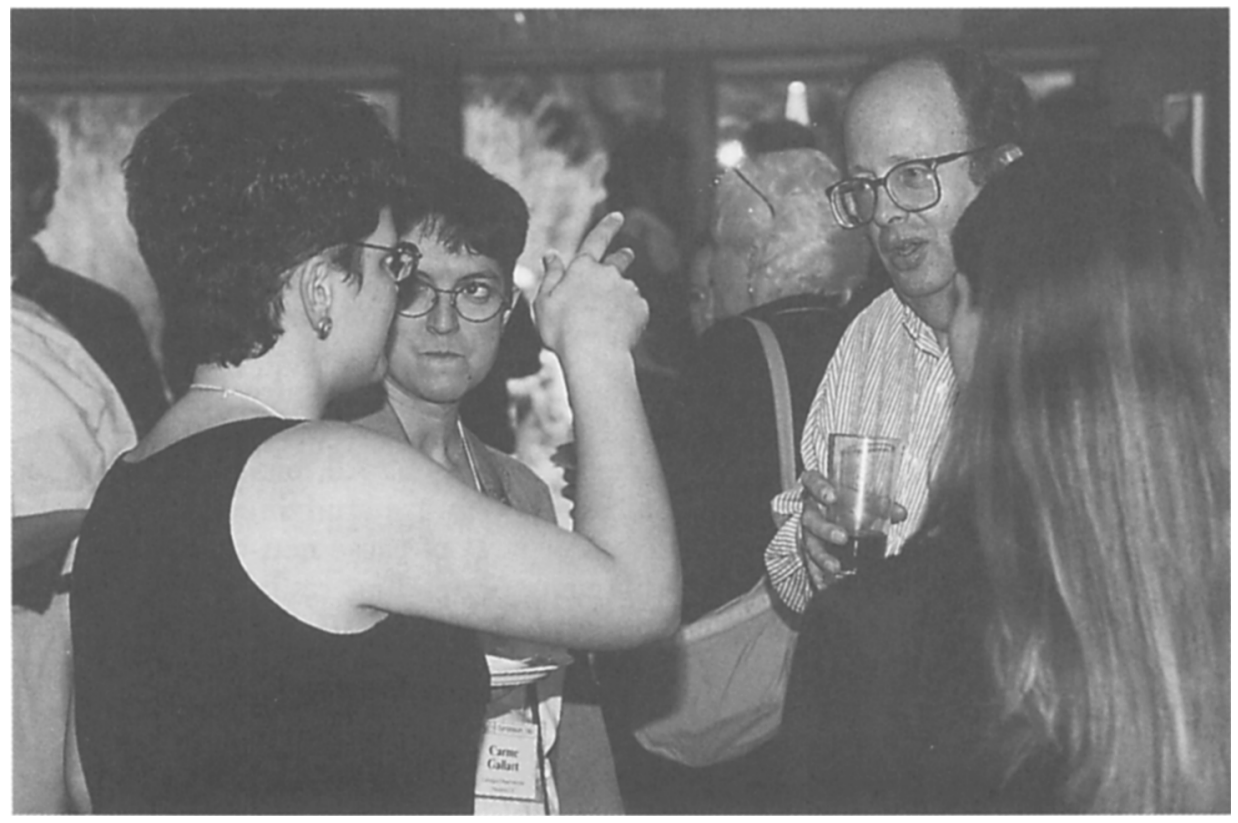

Despina Hatzidimitriou appears to provoke a skeptical reaction from Carme Gallart and Jay Gallagher during the reception. 